\title{
Erythrosine Incorporated Fast-Dissolving Patches for Dental Plaque Disclosing
}

\author{
Prasopchai Tonglairoum ${ }^{1}$, Theerasak Rojanarata ${ }^{1}$, Tanasait Ngawhirunpat ${ }^{1}$, \\ Prasert Akkaramongkolporn ${ }^{1}$, Ruchadaporn Kaomongkolgit ${ }^{2}$, Praneet Opanasopit ${ }^{1, *}$ \\ ${ }^{1}$ Faculty of Pharmacy, Silpakorn University, Nakhon Pathom, Thailand \\ ${ }^{2}$ Department of Oral Diagnosis, Faculty of Dentistry, Naresuan University, Phitsanulok, Thailand
}

Copyright $\subset 2017$ by authors, all rights reserved. Authors agree that this article remains permanently open access under the terms of the Creative Commons Attribution License 4.0 International License

\begin{abstract}
The aim of this study was to develop erythrosine-loaded electrospun polyvinyl pyrrolidone/ hydroxypropyl- $\beta$-cyclodextrin (PVP/HP $\beta C D)$ fiber patches for plaque disclosing. The fiber patches had been fabricated via electrospinning process and were characterized using scanning electron microscope (SEM), Fourier transform infrared spectrophotometry (FT-IR), differential scanning calorimeter (DSC) and powder X-ray diffractometry (PXRD). The fiber patches were then further evaluated for wetting and integration abilities, mechanical properties, loading efficiency, loading capacity and release characteristic. These erythrosine-loaded fiber patches exhibited excellent wetting and disintegration properties and prompt released the erythrosine form the patches after contacted with saliva. These erythrosine-loaded fiber patches could be exploited as a plaque-disclosing material. However, further in vivo studies are needed for the application.
\end{abstract} Keywords Erythrosine, PVP/HP $\beta C D, \quad$ Plaque,
Disclosing Agent, Oral Patches

\section{Background}

Dental plaque is a biofilm composed of a complex microbial community [1-2]. Besides the microorganism, dental plaque consists of carbohydrates and proteins which act as reservoir of nutrient and promote the adherence of the biofilm to the enamel. The metabolic processes from bacteria in the plaque can cause a decrease in the $\mathrm{pH}$ value of the plaque leading to dental carries, periodontal diseases and gingivitis [3]. The plaque cannot be flushed away by simply rinsing with water, and effective elimination of the plaque is necessary for good oral hygiene [4]. Most people do not recognize the quantity and the location of the plaque existing in the mouth because dental plaque is usually transparent, colorless and not easily visible. Recognition of this difficulty has led to the launch of disclosing agents [5]. The plaque disclosing agents color the plaque and make the plaque visible. The use of disclosing agent encourages an individual in the early removal of dental plaque by showing the presence and quantity of plaque [4, 5]. Erythrosine, a water-soluble dye, has been widely used to detect the plaque.

Due to an increasing interest in nanotechnology, nanofibers have obtained lots of attention owing to their marvelous properties. Electrospinning is found to be a versatile and cost-efficient method to fabricate multi-functional nanofibers from numerous polymers with the outstanding properties [6-9]. The nanofibers generated using this technique offer various advantages such as high surface area to volume ratio, tunable porosity and the ability to manipulate nanofibers composition in order to get desired properties and function [10]. Moreover, electrospun nanofibers exhibit high loading capacity, high encapsulation efficiency, and provide coincident delivery of various compounds [11-12]. Because of these attractive properties, electrospun nanofibers have been used in biomedical sciences, filtration, optical sensors and affinity membranes [13-15]

Recently, new strategies for drug delivery have gained much interest. The great advantages of fast-dissolving drug delivery systems; for instance, enhanced drug solubility, onset of action and bioavailability render this system more attractive [16-17]. However, the selection of suitable polymer that can dissolve quickly and easily is essential to achieve the fast-dissolution. Polyvinylpyrrolidone (PVP) is one of the desirable polymer that can provide these features; it is a hydrophilic polymer which can dissolve rapidly and easily in water leading to ultrafast dissolution. This property makes it suitable for oral fast-disintegration formulations [18]. Cyclodextrin (CDs) are cyclic oligosaccharides consisting of 1,4-linked glucopyranoside units having either six, seven, or eight glucose units arranged in a cyclic structure with a hydrophilic outer surface and a lipophilic central cavity which suit for a variety of lipophilic drugs [19-20]. The physicochemical properties of the drug-CDs 
inclusion complexes can be improved. Therefore, the CDs have been utilized in many application fields, such as pharmaceuticals, foods, cosmetics, home/personal care, textiles etc. [21-22]. Previous studies reported that mixing hydroxypropyl- $\beta$-cyclodextrin (HP $\beta C D$ ) with PVP can improve the physical properties of PVP, especially the hygroscopic property of PVP $[18,20]$.

For plaque disclosing purpose, erythrosine is commonly available in the form of solutions that need to be applied on the teeth, or in form of tablets which need to be chewed which is not convenient to use. Therefore, using fast dissolving patches may be more attractive. To best of our knowledge, the preparation of fast-dissolving erythrosine loaded electrospun nanofibers for plaque disclosing has not been reported previously.

Therefore, the aim of this study was to fabricate erythrosine-loaded fast-dissolving fiber patches which exhibit excellent prompt release property for plaque disclosing and may be a potential candidate for plaque disclosing. In this study, erythrosine was selected as a plaque disclosing dye that being incorporated into the PVP/HP $\beta D$ patches. The morphology and physiochemical properties, wetting and integration ability, mechanical properties, loading efficiency, loading capacity, and the release behavior of the fabricate erythrosine-loaded nanofiber patches were investigated.

\section{Methods}

\subsection{Materials}

Polyvinylpyrrolidone (PVP, MW. 1,300,000) was purchased from Sigma-Chemical Co. (St. Louis, MO, USA). 2-hydroxypropyl- $\beta$-cyclodextrin (HP $\beta C D$ ) and erythrosine were provided by Maxway Co. (Bangkok, Thailand) and Nishiki international Co. (Nonthaburi, Thailand), respectively. All other reagents and solvents were of analytical grade and were used without further purification.

\subsection{Fabrication of PVP/HPßCD Patches}

The PVP/HP $\beta C D$ patches were fabricated by electrospinning process using a modified procedure from our previous study [9]. Briefly, the polymer solutions were prepared by dissolving PVP in the 70:20:10 volume ratio of ethanol: water: benzyl alcohol mixture at the concentration of $8 \%(\mathrm{w} / \mathrm{w})$. Then, various amounts of HPßCD $(70-110 \mathrm{mM})$ were added to the polymer solutions. The polymer solutions were then allowed to stir overnight using a magnetic stirrer until homogenous solutions were obtained. Afterwards, the polymer solutions were filled in a 5-ml glass syringe connected to a stainless-steel needle with a $0.9 \mathrm{mM}$ inner diameter. The needle was connected to an emitting electrode with a positive polarity of a Gamma High Voltage Research device. The electrospinning process was performed with a set applied voltage of $15 \mathrm{kV}$, a distance between the tip and the collector of $15 \mathrm{~cm}$, and a feeding rate of $0.4 \mathrm{~mL} / \mathrm{h}$.The electrospun nanofibers were gathered on the surface of an aluminum foil covered the rotating collector.

\subsection{Fabrication of Erythrosine-Loaded PVP/HPßCD Patches}

After obtained the optimal concentration of $\mathrm{HP} \beta C D$ used for the fiber patches preparation, a model dye (erythrosine) at various concentrations $(1 \%, 3 \%$ and $6 \% \mathrm{wt}$ to polymer) was added to the mixture and stirred until the dye was completely dissolved. The viscosity and conductivity of the mixed solutions were determined before the electrospinning process using a Brookfield viscometer (DV-III ultra, Brookfield Engineering Laboratories, USA) and a conductivity meter (Eutech Instruments Pte Ltd, Singapore), respectively. The electrospinning process was conducted with a fixed applied voltage of $15 \mathrm{kV}$, a distance between the tip and the collector of $15 \mathrm{~cm}$, and a feeding rate of $0.4 \mathrm{~mL} / \mathrm{h}$. The electrospun nanofibers were collected on the surface of an aluminum foil covered the rotating collector.

\subsection{Characterization of the Electrospun PVP/HPBCD Patches}

\subsubsection{Scanning Electron Microscope (SEM)}

SEM images of the PVP/HP $\beta C D$ patches were taken using a scanning electron microscope (SEM, Camscan Mx2000, England) in order to observe the morphology and diameter of the PVP/HP $\beta C D$ fibers. In this process, a small piece of the fiber patches was sputtered with a thin layer of gold prior to the SEM observations. To determine the average diameter of the fibers, the images were analyzed using an image analysis software (JMicroVision V.1.2.7, Switzerland).

\subsubsection{Fourier Transform Infrared Spectrophotometry (FT-IR)}

The Fourier transform infrared spectroscopy was carried out using a Fourier transform infrared spectrophotometer (FT-IR, Nicolet 4700, USA) and Omnic software at the wave number range of $400-4000 \mathrm{~cm}^{-1}$. The fiber samples were crushed and pressed into $\mathrm{KBr}$ dishes pellets prior to the analysis.

\subsubsection{Differential Scanning Calorimeter}

The thermal behavior and composition of the PVP/HP $\beta C D$ patches were examined using a differential scanning calorimeter (DSC, Pyris Diamond DSC, PerkinElmer instrument, USA). Samples (5-10 mg) were placed in aluminum sample pans and heated, under a nitrogen atmosphere, at a rate of $10^{\circ} \mathrm{C} / \mathrm{min}$ to $500^{\circ} \mathrm{C}$. 


\subsubsection{Powder X-ray Diffractometry (PXRD)}

The physical state of erythrosine in the fiber patches was investigated by a powder x-ray diffraction analyses (PXRD, Miniflex II, Rigaku, Japan) using Nickel-filtered $\mathrm{Cu}$ radiation generated in a sealed tube operated at $30 \mathrm{kv}$ and $15 \mathrm{~mA}$. The diffraction patterns of the patches were recorded in the $\theta$ range of $5-45^{\circ}$ at $5^{\circ} \mathrm{min}^{-1}$.

\subsubsection{Mechanical Characterizations}

Stress, stain and young's modulus values of the patches were measured using a texture analyzer (TA. XT plus, Stable Micro Systems, UK) by applying a $5 \mathrm{~kg}$ load cell equipped with tensile grip holder. All samples were cut into rectangle shapes with dimensions of $35 \times 6 \mathrm{mM}^{2}$.

\subsection{Wetting Time and Disintegration Time}

The study of wetting time was carried out using a procedure from our previous study (Tonglairoum et al., 2014). Two layers of tissue paper were placed on a Petri dish with a diameter of $10 \mathrm{~cm}$. The PVP/HP $\beta C D$ fiber patches were placed on the paper wetted with artificial saliva (2.38 $\mathrm{g}$

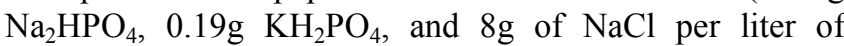
distilled water adjusted with the phosphoric acid to $\mathrm{pH}$ $6.8 \pm 0.05$ ) (Peh et al., 1999), and the excess saliva was completely removed. The time required for water to diffuse from the wetted tissue paper throughout the patches was recorded as the wetting time. To assess the disintegration time, a beaker containing $20 \mathrm{~mL}$ of artificial saliva was employed, and the PVP/HP $\beta C D$ fiber patches were placed in the beaker. The time required for the patches to complete disintegrate or dissolution was recorded as disintegration time. The wetting and disintegration processes were recorded using a digital video recorder (Cannon, Japan).

\subsection{Loading Efficiency and Loading Capacity}

The quantity of erythrosine contained in the fiber patches were quantified using a UV-visible spectrophotometer (Agilent G1103A UV-Vis Spectrophotometer, Agilent Technologies, USA) at $530 \mathrm{~nm}$. Briefly, precisely weighed samples $(2 \mathrm{mg})$ of the fiber patches were dissolved in $10 \mathrm{~mL}$ of deionized water and stirred using a magnetic stirrer for $24 \mathrm{~h}$. The experiments were performed in triplicate. The loading efficiency and loading capacity were calculated according to equation (1) and (2), respectively.

$$
\% \text { loading efficiency }=\left(\mathrm{E}_{\mathrm{f}} / \mathrm{E}_{\mathrm{i}}\right) \times 100
$$

where $E_{f}$ is the amount of erythrosine contained in the fiber patches and $E_{i}$ is the initial amount of erythrosine (from the feeding solution).

$$
\text { Loading capacity }=\left(\mathrm{E}_{\mathrm{f}} / \mathrm{M}\right)
$$

where $E_{f}$ is the amount of erythrosine contained in the fiber patches and $\mathrm{M}$ is the weight of the fiber patches.

\subsection{In vitro Release Studies}

The in vitro release studies of erythrosine from the fiber patches were carried out in artificial saliva. Briefly, $10 \mathrm{mg}$ of the erythrosine loaded fiber patches was submerged in a $50 \mathrm{~mL}$ bottle containing $10 \mathrm{~mL}$ of artificial saliva ( $\mathrm{pH} 6.8$ ) that was incubated at $37^{\circ} \mathrm{C}$ and shaken at $150 \mathrm{rpm}$. At given intervals, $1.0 \mathrm{~mL}$ aliquots of the release medium were withdrawn and replaced with fresh medium to maintain a constant volume. The released erythrosine in each aliquot was determined using a UV-visible spectrophotometer (Agilent G1103A UV-Vis Spectrophotometer, Agilent Technologies, USA). The experiments were conducted in triplicate.

\section{Results and Discussion}

\subsection{Fabrication of PVP/HPßCD Patches}

To fabricate the fiber patches, PVP was exploited as a fiber forming polymer. $\mathrm{HP} \beta \mathrm{CD}$ was added to the polymer solution in order to lessen the hygroscopic property of PVP and increase the stability of the fibers and the erythrosine. To obtain the smooth and homogenous fibers, the concentration of $\mathrm{HP} \beta C D$ in the polymer solution was varied from 70 to $110 \mathrm{mM}$. The diameter of the patches comprising different amounts of HP $\beta C D$ was in the range of $333-363 \mathrm{~nm}$. Increasing the HP $\beta C D$ concentration resulted in an increase in fiber diameters. The fiber diameter increased from 333.67 to 334.46 and $363.69 \mathrm{~nm}$ as the HP $\beta C D$ concentration was increased from $70 \mathrm{mM}$ to 90 and $110 \mathrm{mM}$, respectively. The increase in fiber diameter may be due to the increase in the viscosity of the spinning solution after adding HP $\beta C D$ (data not shown). Fig.la-c shows the SEM images of the electrospun PVP/HP $\beta C D$ fiber patches containing different amounts of HP $\beta C D$. The smooth nano-sized fibers with some beads on the fibers were obtained from the polymer solution containing $70 \mathrm{mM} H \mathrm{HP} \beta C D$. After increased the $\mathrm{HP} \beta C D$ concentration to $90 \mathrm{mM}$, the smooth and homogeneous fibers without beads were obtained. The beads reappeared after increased the $\mathrm{HP} \beta C D$ concentration to $110 \mathrm{mM}$. Solution properties have been found to affect the morphology of the fiber patches. Generally, the fiber diameter and the bead formation are affected by the solution viscosity which is one of the major parameters influencing the fiber diameter. Generally, the viscosity of the solution is related to the extent of polymer molecule chain entanglement within the solution. A higher viscosity results in a large fiber diameter [23]. 

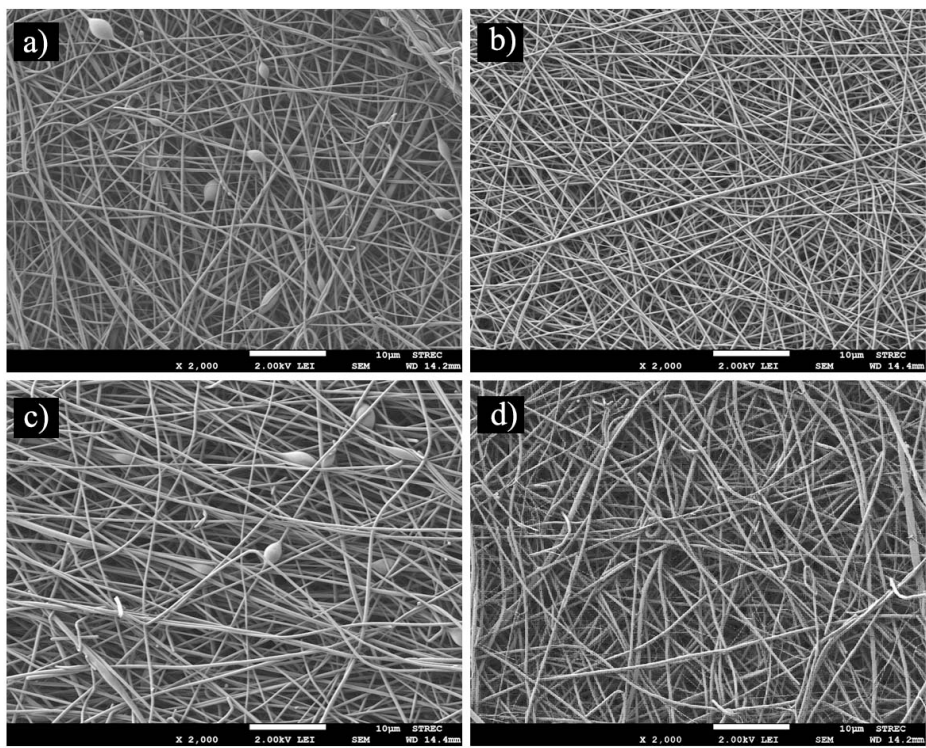

Figure 1. The SEM image (1,000x) of the PVP/HP $\beta C D$ fiber patches electrospun from the polymer solution containing different HP $\beta C D$ concentrations: a) $70 \mathrm{mM}$, b) $90 \mathrm{mM}$, c) $110 \mathrm{mM}$ d) $90 \mathrm{mM}$ and $6 \%$ erythrosine.

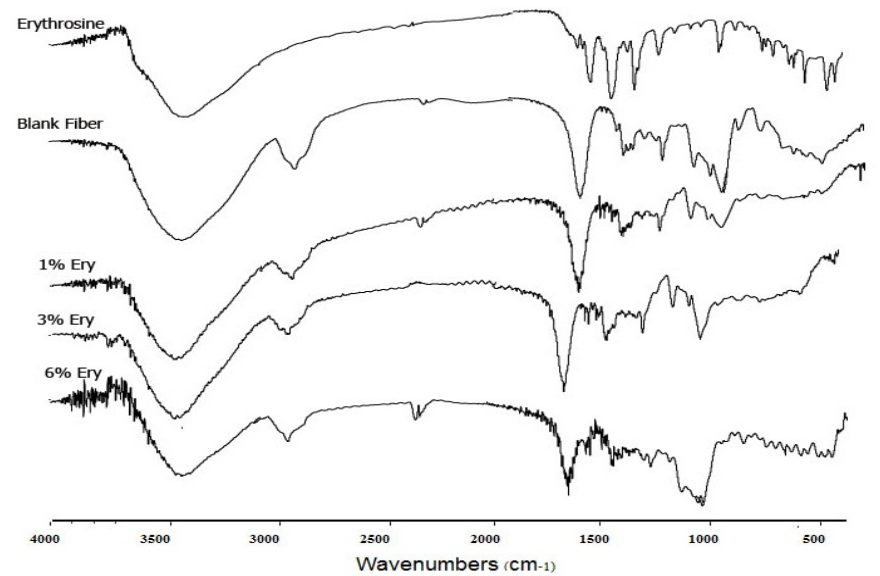

Figure 2. FT-IR spectra of erythrosine and the blank PVP/HP $\beta C D$ fiber patches containing $0 \%, 1 \% 3 \%$ and $6 \%$ erythrosine.

In addition, viscosity has a significant effect on whether the electrospinning jet breaks up into small droplets or whether the resulting electrospun fibers contain beads [11]. Therefore, the optimal concentration of HP $\beta C D$ used in the polymer solution was $90 \mathrm{mM}$, and this $\mathrm{HP} \beta C D$ concentration was selected for the preparation of erythrosine loaded PVP/ $\mathrm{HP} \beta C D$ patches. Erythrosine was incorporated into the fiber patches at the varied concentration of $1-6 \%$, and the maximum concentration of erythrosine that could be incorporated in the patches without any effects on the morphology of the patches was found to be $6 \%$. The fiber morphology of $6 \%$ (weight to polymer) erythrosine loaded PVP/ HP $\beta C D$ patches is presented in Fig.1d. After erythrosine was added, the fiber diameter increased to $427.6 \mathrm{~nm}$. The increase in diameter was due to the increase in viscosity of the polymer solution after erythrosine was added.

\subsection{Fourier Transform Infrared Spectrophotometry (FT-IR)}

The incorporation of erythrosine into the fiber patches was confirmed by FT-IR analysis. The FTIR spectra of erythrosine powder, the blank fiber patches and 1-6\% erythrosine-loaded fiber patches are displayed in Fig. 2. The spectrum of the blank PVP/HP $\beta C D$ fiber patches displayed absorption peaks at 3417,2936 and $1032 \mathrm{~cm}^{-1}$ that corresponds to the stretching vibrations of $\mathrm{N}-\mathrm{H}, \mathrm{O}-\mathrm{H}, \mathrm{C}-\mathrm{H}$ and C-O stretching vibration respectively. Erythrosine powder exhibited absorption bands at 1602,1541 , and $1455 \mathrm{~cm}^{-1}$ that were attributed to the benzene ring stretches. The peak at $963 \mathrm{~cm}^{-1}$ was assigned to the $\mathrm{C}=\mathrm{C}-\mathrm{H}$ functional group. The dominant bands presented in the erythrosine powder spectrum were also observed in the spectra of the $1-6 \%$ erythrosine-loaded fiber patches. These findings confirm the successful incorporation of erythrosine into the fiber patches. 


\subsection{Differential Scanning Calorimeter (DSC)}

The thermal behaviors of the blank fiber patch and the erythrosine loaded fiber patches were investigated using a DSC and the DSC thermograms are shown in Fig.3. The DSC thermogram of erythrosine exhibited an endothermic peak at $208.93^{\circ} \mathrm{C}$ due to its melting temperature. The endothermic curves of the fiber patches showed no significant changes in the thermal properties after erythrosine was incorporated in the fiber patches when concentration of erythrosine was increased to 1,3 and $6 \%$, respectively. This indicates that erythrosine loaded in the fiber patches does not affect the thermal behavior of the nanofibers.

\subsection{X-ray Diffractometry (XPRD)}

The molecular state of erythrosine dispersed in the fiber patches was determined, and the diffractograms are presented in Fig.4. The diffractogram of erythrosine exhibited dominant crystalline peaks, signifying its high degree of crystallinity. However, no such peak was found in the diffractograms of the fiber patches after erythrosine was loaded into the fiber patches suggesting that the erythrosine was incorporated in the fiber patches in amorphous form.

\subsection{Mechanical Testing}

The Young's modulus values of all tested fiber patches were investigated. The Young's modulus of the blank fibers was $0.29 \mathrm{MPa}$, and the value rose to $1.38,1.49$ and $1.60 \mathrm{MPa}$ after $1 \%, 3 \%$ or $6 \%$ erythrosine, respectively, was incorporated into the fiber patches. Adding erythrosine into the fiber patches did not show any negative effects on the tensile strength of the fiber patches, but it improved the mechanical properties of the patches. This may be due to the change in fiber properties after erythrosine was incorporated into the nanofiber patches. However, there are many factors that could affect the mechanical properties of the patches. All factors should be taken into account to determine the actual effect.

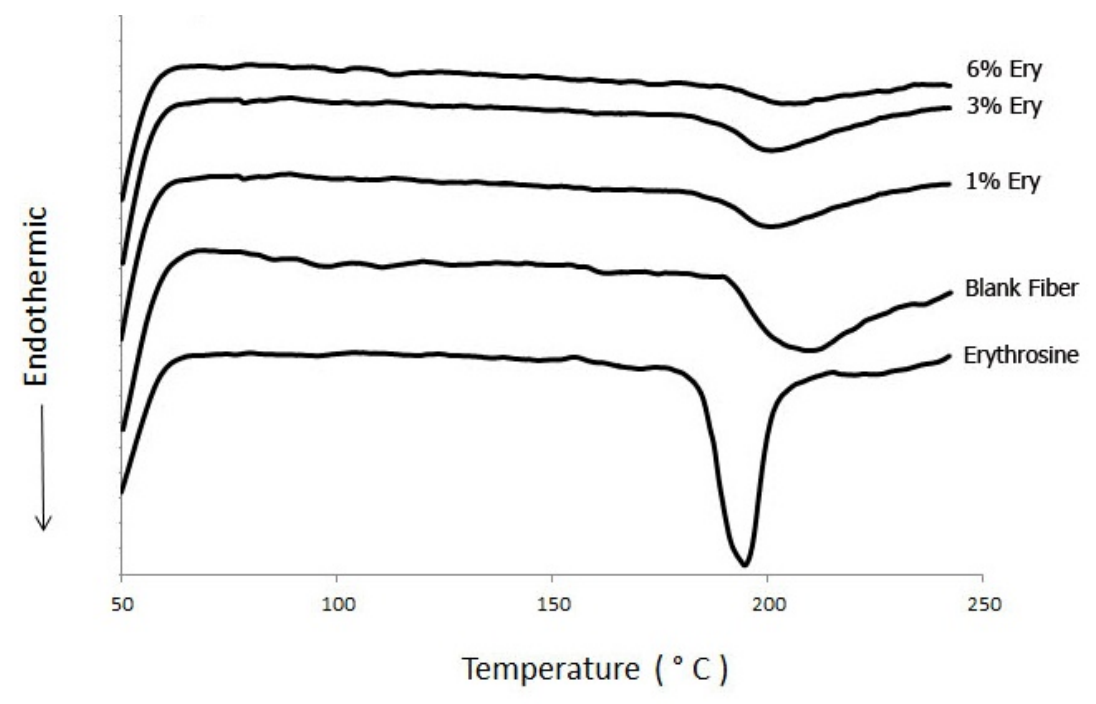

Figure 3. DSC thermogram of erythrosine and the blank PVP/HP $\beta C D$ fiber patches containing $0 \%, 1 \% 3 \%$ and $6 \%$ erythrosine

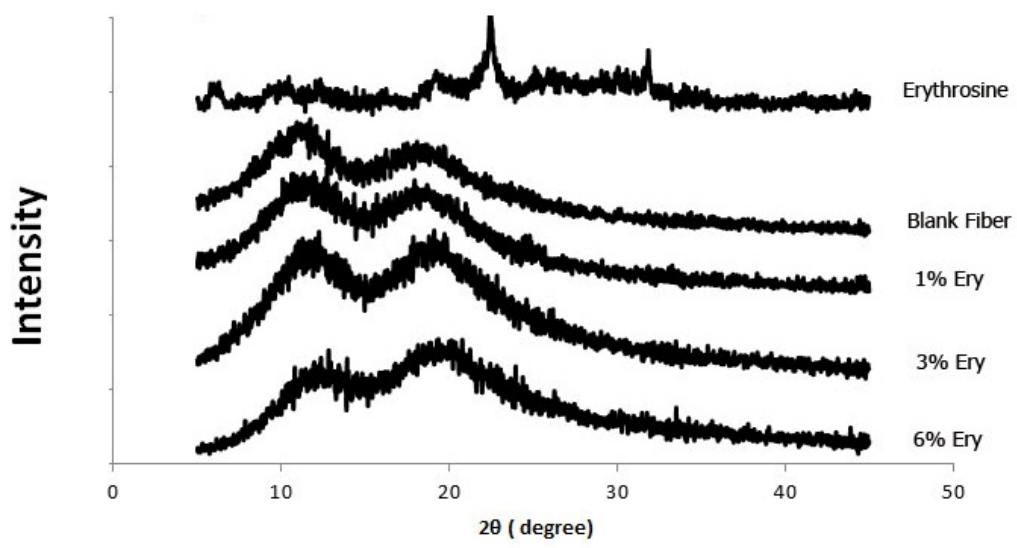

Figure 4. X-ray diffraction pattern of erythrosine and the blank PVP/HP $\beta C D$ fiber patches containing $0 \%, 1 \% 3 \%$ and $6 \%$ erythrosine 


\subsection{Wetting Time and Disintegration Time}

The wetting and disintegration abilities of the patches were assessed, and the results are presented in Fig. 5 and Fig. 6 . The blank fibers were completely wet and lost its original white color within $2 \mathrm{~s}$. The wetting time for $1 \%, 3 \%$ and $6 \%$ erythrosine loaded fiber patches were $1.67,2.33$ and $2.67 \mathrm{~s}$, respectively. For the disintegration study, the fiber patches were placed in a glass of artificial saliva. All the fiber patches exhibited rapid disintegration. The disintegration time was found to be less than $1 \mathrm{~s}$. The excellent wetting ability and disintegration of the patches may be due to the highly porous structure of the fiber patches that allowed rapid penetration of saliva throughout the fibers.

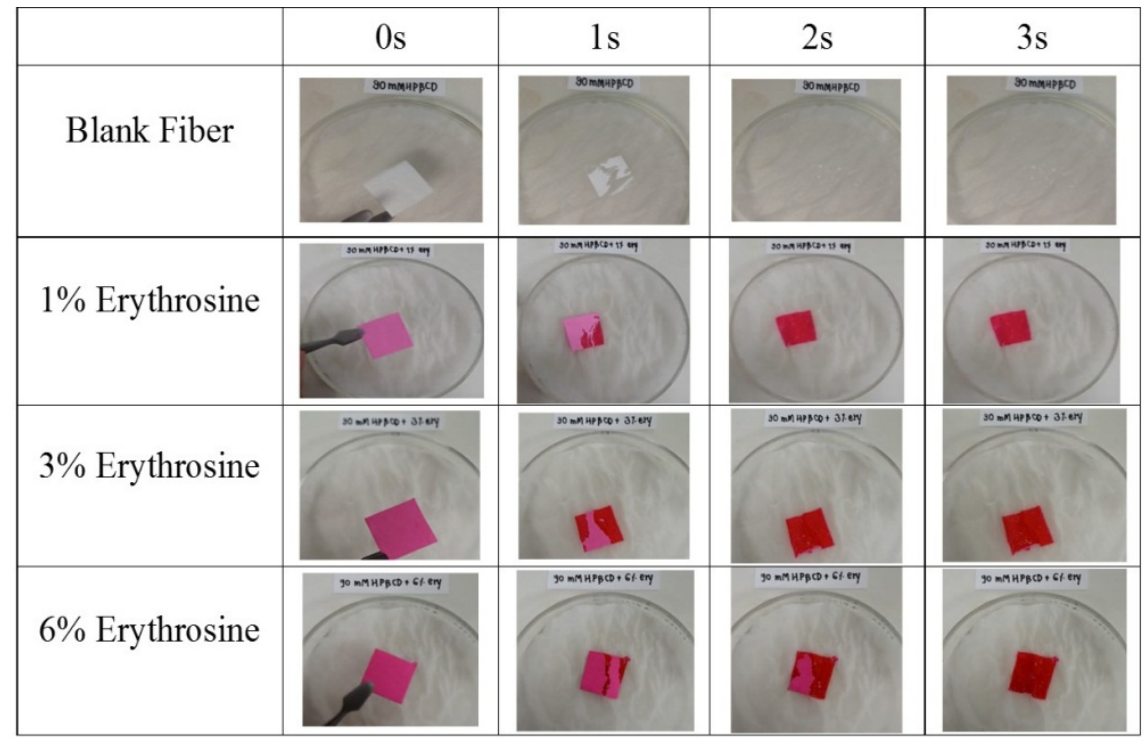

Figure 5. Wetting ability of the fiber patches containing $0-6 \%$ erythrosine

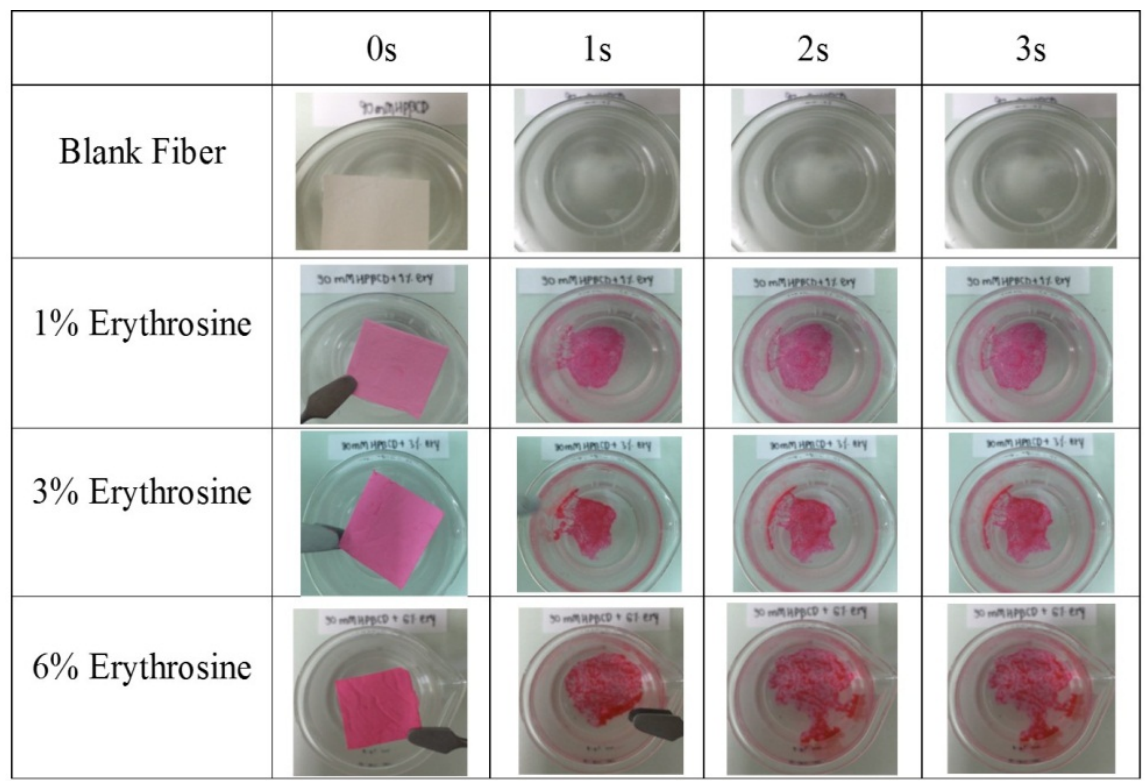

Figure 6. disintegration of the fiber patches containing 0-6\% erythrosine 


\subsection{Loading Efficiency and Loading Capacity}

Erythrosine was incorporated into the nanofibers using electrospinning process. During the electrospinning process, the solvent was rapidly removed during elongation of the jet and the drug was embedded in the dried nanofibers. The schemes illustrated the drug loading into the nanofibers by electrospinning process and the release of the drug from the nanofibers is presented in Fig.7. The loading efficiency and loading capacity of erythrosine in the erythrosine-loaded fiber patches were determined, and the results are listed in Table 1. Erythrosine was loaded into the patches with high loading efficiency (>90\%). Increasing the initial amount of erythrosine in the polymer solutions resulted in the increase in loading capacity. This may be because the maximum capacity of nanofiber mats to carry the drug was not reached even at the highest amount $(6 \%)$ of erythrosine being loaded in the nanofibers (in this study).

\subsection{Release Study}

The release characteristics of erythrosine from the fiber patches in artificial saliva were assessed, and the results are presented in Fig.8. Prompt release of erythrosine form the patches had been observed after the patches were contacted with saliva. This is due to the extremely high surface area and porous structure of the electrospun fiber patches which promoted a remarkably fast release [18]. Moreover, the hydrophilic property of PVP could diminish the wetting time of the patches. As it can be seen from Fig.7, approximate $90 \%$ of the erythrosine contained in the patches released to the release medium within $15 \mathrm{~s}$. No significant difference had been observed in the $\%$ cumulative release of erythrosine form the patches containing different amounts of erythrosine.
This is because erythrosine was incorporated into the nanofibers in an amorphous form which is able to dissolve easily and quickly when contact with water.

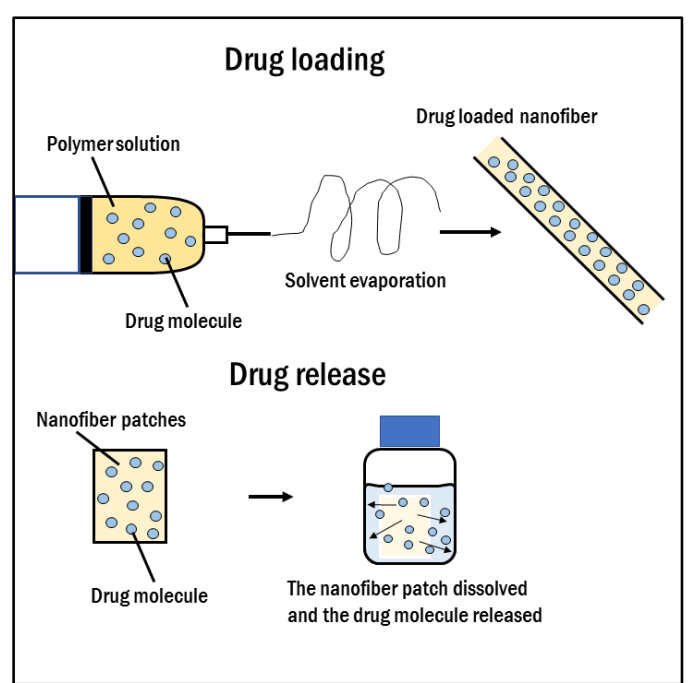

Figure 7. Illustration of erythrosine loading into the nanofibers by electrospinning process and the release of the drug from the nanofibers

Table 1. Loading efficiency and loading capacity of erythrosine in the fiber patches

\begin{tabular}{|c|c|c|}
\hline$\%$ Erythrosine & Loading efficiency (\%) & Loading capacity (\%) \\
\hline $1 \%$ & $98.89 \pm 3.48$ & $1.09 \pm 0.03$ \\
\hline $3 \%$ & $96.31 \pm 6.76$ & $3.19 \pm 0.20$ \\
\hline $6 \%$ & $95.01 \pm 7.14$ & $5.70 \pm 0.43$ \\
\hline
\end{tabular}

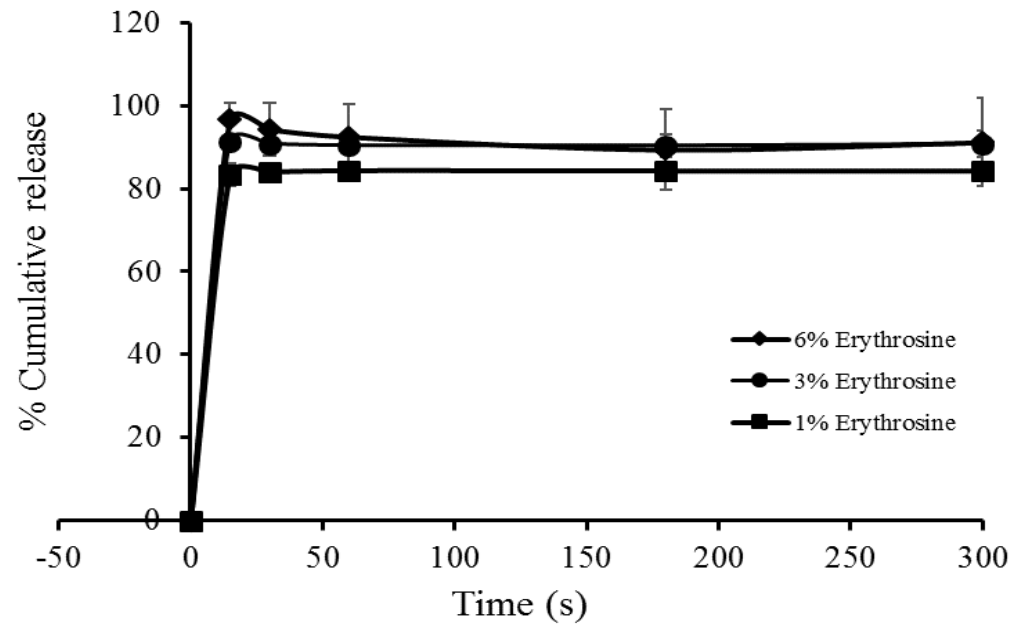

Figure 8. Release characteristics of erythrosine from the fiber patches containing different amounts of erythrosine 


\section{Conclusions}

The fast-dissolving PVP-HP $\beta C D$ electrospun fiber patches containing erythrosine were successfully fabricated using electrospinning process. Erythrosine could be incorporated at the maximum content of $6 \%$ (wt to polymers). The erythrosine loaded fiber patches exhibited very fast release and could release more than $85 \%$ of the erythrosine contained in the patches within $15 \mathrm{~s}$. These erythrosine-loaded fiber patches could be used as a plaque disclosing materials. However, further in vivo studies are needed for the application.

\section{Acknowledgments}

The authors would like to thank Faculty of Pharmacy, Silpakorn University for their financial support.

\section{REFERENCES}

[1] Marsh PD. Dental plaque: biological significance of a biofilm and community life-style. J Clin Periodontol. 2005; 32(6): $7-15$.

[2] Seneviratne CJ, Zhang CF, Samaranayake LP. Dental plaque biofilm in oral health and disease. Chin J Dent Res, 2011; 14(2): 87-94.

[3] Sbordone L, Bortolaia C. Oral microbial biofilms and plaque-related diseases: microbial communities and their role in the shift from oral health to disease. Clin Oral Investig, 2003; 7(4): 181-188

[4] Chowdhary Z, Mohan R, Sharma V, Rai R, Das A. Disclosing agents in periodontics: and update. Journal of Dental College Azamgarh, 2015; 1(1):103-110.

[5] Gillings BRD. Recent developments in dental plaque disclosants. Aust Dent J. 1976; 22(4): 260-266.

[6] Bai J, Yang Q, Li M, Zhang C, Li Y. Synthesis of poly (N-vinylpyrrolidone)/ $\beta$-cyclodextrin composite nanofibers using electrospinning techniques, J Mater Process Technol. 2008; 208: 251-254.

[7] Bai J, Li Y, Li M, Wang S, Zhang C, Yang Q. Electrospinning method for the preparation of silver chloride nanoparticles in PVP nanofiber. Appl Surf Sci. 2008; 254: 4520-4523.

[8] Reneker DH, Chun I. Nanometer diameter fibers of polymer produced by electrospinning. Nanotechnology. 1996; 7: 216-223.

[9] Uyar T, Besenbacher F. Electrospinning of cyclodextrin functionalized polyethylene oxide (PEO) nanofibers. Eur Polym J. 2009; 45: 1032-1037.
[10] Sill TJ, Von Recum HA. Electrospinning: Applications in drug delivery and tissue engineering. Biomaterials. 2008; 29:1989-2006.

[11] Arthanari S, Mani G, Jang JH, Choi JO, Cho YH, Lee JH, et al. Preparation and characterization of gatifloxacin-loaded alginate/poly (vinyl alcohol) electrospun nanofibers. Artif Cells Nanomed Biotechnol. 2016; 44(3):847-52.

[12] $\mathrm{Hu} \mathrm{X}$, Liu S, Zhou $\mathrm{G}$, Huang $\mathrm{Y}$, Xie Z, Jing $\mathrm{X}$. Electrospinning of polymeric nanofibers for drug delivery applications. J Control Release, 2014; 185(0): 12-21.

[13] Bamford CH, Al-Lamee KG, Pm-brick MD, Wear TJ. Studies of a novel membrane for affinity separations. I. Functionalisation and protein coupling, J. Chromatogr. 1992; 606: 19-31.

[14] Ongun MZ, Ertekin K, Gocmenturk M, Ergun Y, Suslu A. Copper ion sensing with fluorescent electrospun nanofibers. Spectrochim Acta A Mol Biomol Spectrosc. 2012; 90: 177-85.

[15] Charernsriwilaiwat N, Opanasopit P, Rojanarata T, Ngawhirunpat T. Lysozyme-loaded, electrospun chitosan-based nanofiber mats for wound healing. Int J Pharm. 2012; 427: 379- 84 .

[16] Arkash V, Maan S, Deepika, Yadav SK, Hemlata, Jogpal V. Fast disintegrating tablets: Opportunity in drug delivery system. J Adv Pharm Tech Res. 2011;2(4):223-35.

[17] Li X, Kanjwa MA, Lin L, Chronakis IS. Electrospun polyvinyl-alcohol nanofibers as oral fast-dissolving delivery system of caffeine and riboflavin. Colloids Surf B. 2013; 103:182-188.

[18] Tonglairoum P, Ngawhirunpat $T$, Rojanarata $T$, Kaomongkolgit R, Opanasopit P. Fast-acting clotrimazole composited PVP/HPbetaCD nanofibers for oral candidiasis application. Pharm Res. 2014;31(8):1893-906.

[19] Tiwari G, Tiwari R, Rai AK. Cyclodextrins in delivery systems: J Pharm Bioallied Sci. 2010;2(2):72-79.

[20] Tonglairoum P, Chuchote T, Ngawhirunpat T, Rojanarata T, Opanasopit P. Encapsulation of plai oil/2-hydroxypropyl-beta-cyclodextrin inclusion complexes in polyvinylpyrrolidone (PVP) electrospun nanofibers for topical application. Pharm Dev Technol. 2014; 19(4):430-7.

[21] Valle D, Martin EM. Cyclodextrins and their uses: a review, Process Biochem. 2004; 39:1033-1046.

[22] Uslu I, Keskin S, Gül A, Karabulut TC, AksumL. Preparation and Properties of electrospun poly (vinyl alcohol) blended hybrid polymer with aloe vera and HPMC as wound dressing. Hacettepe J Biol \& Chem. 2010; 38: 19-25.

[23] Ramakrishna S, Fujihara K, Teo WE, Lim TC, Ma Z. An introduction to electrospinning and nanofibers, New Jersey: World Scientific, 1st Ed. 2005. pp. 91-101. 